\title{
Exciting Turbulence with Polymers
}

\section{The addition of polymers to fluids leads to the emergence of a new kind of turbulence in fluid jets-as revealed by high-speed imaging experiments.}

\author{
By Michael D. Graham
}

$\prod$ urbulence-a fluid flow characterized by chaotic vortices-arises when the inertia of the fluid dominates over its viscosity. Turbulence can be bad when it increases energy consumption of airplanes but good when it allows us to quickly mix cream into our coffee. Finding ways to modify turbulence to suit the situation at hand is a long-standing research topic. One approach is to add tiny amounts of long polymer molecules, which can suppress the natural mechanisms driving turbulence, thereby reducing energy dissipation [1]. Researchers have recently begun to appreciate that these additives may not only suppress turbulence but may also lead to qualitatively new mechanisms for producing turbulence. Now, Sami Yamani of the Massachusetts Institute of Technology and colleagues illustrate this point in an important context-a jet of fluid fired into a background fluid. Using high-speed imaging, the researchers investigate the novel flow structures induced by the addition of polymers to the jet solution. By observing jets with different polymer concentrations and molecular weights, they demonstrate that such flow structures have features that signal the emergence of a fundamentally new type of turbulence [2].

Fluid jets are used in many applications, especially for mixing fluids together-inject a syringe full of dyed water into a sink full of clear water and you'll see why. Why do jets induce mixing? To a first approximation, a jet is a cylinder of "inner" fluid moving in an "outer" fluid-the surrounding liquid. If a perturbation causes the jet cylinder to pinch inward, the fluid will move faster in the pinch than it does elsewhere because the same amount of fluid is passing through a narrower region, just as a river runs faster where it is shallow. According to Bernoulli's principle, pressure decreases where velocity increases, so the lower pressure in the pinch will draw the outer fluid inward, narrowing the pinch and decreasing the pressure further. This basic instability drives further movement of the outer fluid into the jet, causing turbulent vortices that speed up the mixing [3].

This picture can change in nontrivial ways if polymer molecules are added to the jet. A long, linear polymer molecule in solution can be regarded as a tiny, floppy string that is buffeted by the motion of the surrounding solvent [4]. In a still fluid, the Brownian motion of the solvent molecules colliding with the polymer molecules randomizes the orientations of the various parts of the string-a polymer at equilibrium looks like a squiggle called a random coil. In a flowing fluid, the fluid motion straightens out the strings, while Brownian motion still works to randomize them. The competition of these two effects leads to forces between the polymers and the solvent that give the fluid some "elasticity" - the ability to recover the original conformation when deformed. This ability arises because stretched polymer molecules relax back to their squiggly equilibrium conformations on a timescale that scales inversely with polymer length.

This picture implies that in simple, uniform flows, the polymers simply act to resist fluid motion, making the viscosity, or resistance to deformation, of a polymer solution higher than that of the solvent alone. The fun begins when flows are nonuniform so that the molecules are stretched differently in different parts of the fluid. These nonuniformities can lead to imbalances in polymer forces that can suppress fluid motion but also drive new instabilities [5]. For a polymer solution in a straight channel, experiments and computations reveal that at low elasticity, the former effect dominates: By resisting stretching, polymer molecules suppress the turbulent vortices that form near the channel walls [6]. The suppression of these 


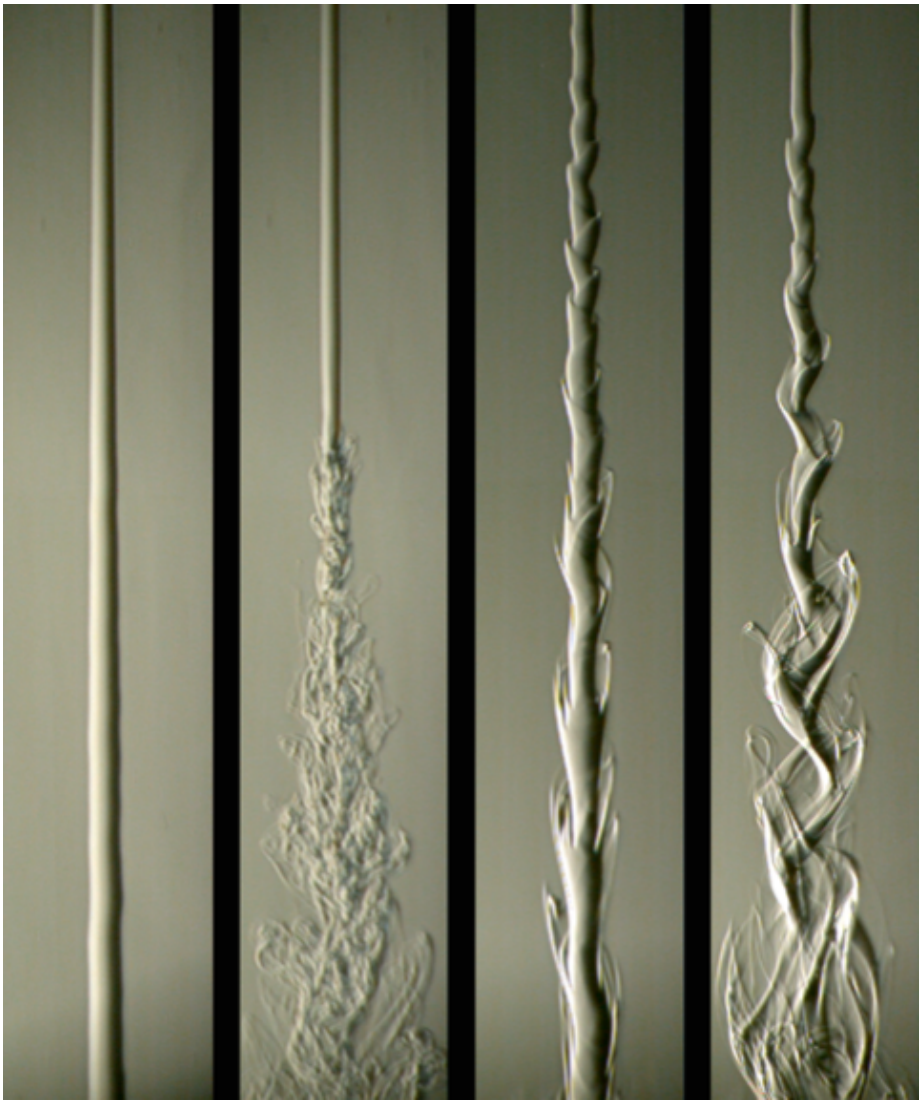

Figure 1: High-speed images of a submerged fluid jet reveal different flow regimes that can be induced by the addition of polymers to the flow [2]. From left to right: laminar flow; Newtonian turbulence; elastoinertial filaments; elastoinertial turbulence.

Credit: S. Yamani et al. [2]

vortices, whose axes of rotation are aligned with the flow, leads to drag reduction. But for higher elasticity, the presence of polymers is destabilizing, and a form of turbulence with very different features, dubbed elastoinertial turbulence (EIT), emerges [7, 8]. In EIT, the vortices are slower and have axes that are perpendicular, rather than parallel, to the flow direction. For all fluid types, the polymer length is the dominant factor in determining how much the polymer affects the flow-the longer the polymer, the larger the drag reduction-while the specific polymer used (such as polyethylene oxide, polyacrylamide, aloe vera, or DNA) isn't as important.

Yamani and co-workers explore the interplay between inertia and elasticity by performing imaging experiments on aqueous solutions of polyethylene oxide (PEO) injected into water. By using different concentrations and molecular weights of PEO, they cover a broad parameter range and lay out a detailed map of how the jet evolves as they tweak the relative magnitudes of inertia, viscosity, relaxation time, and polymer concentration (Fig. 1).

Several striking observations emerge. Even at very low polymer concentrations, the jet turbulence changes dramatically. The flow transitions from smooth, or "laminar," to turbulent at a substantially reduced jet velocity, and the resulting turbulence is visually very different from the simple-fluid case. Specifically, the jet exhibits a much more filamentous structure. At higher concentrations, the turbulent dynamics of the jet are suppressed-producing a new regime in which the fluctuations of the jet's surface grow into filaments, while the jet core remains coherent (see the third panel of Fig. 1).

Another important observation is that this turbulence displays different scaling laws than those found in simple fluids such as water or air. A universal feature of turbulent dynamics in these simple fluids is the scaling of fluctuation energy with the $-5 / 3$ power of the fluctuation frequency. Theory has shown that power laws generally emerge in systems that are self-similar. For turbulence, the self-similarity is that of the turbulent eddies at different spatial scales [9]. The researchers find that their polymer jets also obey a power law, which points at self-similarity but with a different exponent of -3 . Performing an analysis similar to one previously performed [10], the team concludes that this value arises because the polymers prevent the fluid from stretching at a rate larger than the relaxation rate of the polymers. The different scaling behavior thus indicates the presence of a new kind of turbulence-one that's supported by different microscopic mechanisms.

Polymers like PEO are added to fluids in applications including the formation of gels, the control of misting in sprays, and the reduction of water levels in irrigation canals [11]. But our understanding of the flow of these polymer solutions still leaves much to be desired, especially in the already complex regime of turbulent flows. The filamentous structures found by Yamani and colleagues may be related to the sheet-like structures predicted to arise in EIT of channel flows [7]. But the jet geometry may offer a more convenient experimental platform 
for visualizing the detailed flow structures and studying their connections to the scaling laws of turbulence. More broadly, this work will motivate further study of the fascinating interactions between polymer dynamics and flow and perhaps inspire new, unexpected applications.

Michael D. Graham: Department of Chemical and Biological

Engineering, University of Wisconsin-Madison, Madison, WI, USA

\section{REFERENCES}

1. C. M. White and M. G. Mungal, "Mechanics and prediction of turbulent drag reduction with polymer additives," Annu. Rev. Fluid Mech. 40, 235 (2008).

2. S. Yamani et al., "Spectral universality of elastoinertial turbulence," Phys. Rev. Lett. 127, 074501 (2021).

3. M. L. Norman and K.-H. A. Winkler, Supersonic jets, Los Alamos Science Spring/Summer 1985, No. 12, 38 (1985).

4. M. D. Graham, Microhydrodynamics, Brownian Motion, and Complex Fluids (Cambridge University Press, Cambridge, 2018).

5. E. S. G. Shaqfeh, "Purely elastic instabilities in viscometric flows," Annu. Rev. Fluid Mech. 28, 129 (1996).

6. W. Li and M. D. Graham, "Polymer induced drag reduction in exact coherent structures of plane Poiseuille flow," Phys. Fluids 19, 083101 (2007).

7. D. Samanta et al., "Elasto-inertial turbulence," Proc. Natl. Acad. Sci. U.S.A. 110, 10557 (2013); A. Shekar et al., "Critical-layer structures and mechanisms in elastoinertial turbulence," Phys. Rev. Lett. 122, 124503 (2019).

8. G. H. Choueiri et al., "Exceeding the asymptotic limit of polymer drag reduction,” Phys. Rev. Lett. 120, 124501 (2018).

9. U. Frisch, Turbulence: The Legacy of A. N. Kolmogorov (Cambridge University Press, Cambridge, 1995).

10. E. J. Hinch, "Mechanical models of dilute polymer solutions in strong flows," Phys. Fluids 20, S22 (1977); R. Vonlanthen and P. A. Monkewitz, "Grid turbulence in dilute polymer solutions: PEO in water," J. Fluid Mech. 730, 76 (2013).

11. W. Bouchenafa et al., "Water soluble polymers as a means to increase flow capacity: Field experiment of drag reduction by polymer additives in an irrigation canal," J. Hydraul. Eng. 147, 05021003 (2021). 